\title{
Compressed Sensing of EEG with Gabor Dictionary: Effect of Time and Frequency Resolution
}

\author{
Phuong Thi Dao, Anthony Griffin and Xue Jun Li, Senior Member, IEEE
}

\begin{abstract}
Electroencephalogram (EEG) signals have been widely used to analyze brain activities so as to diagnose certain brain-related diseases. They are usually recorded for a fairly long interval with adequate resolution, consequently requiring a considerable amount of memory space for storage and transmission. Recently compressed sensing (CS) has been proposed in order to effectively compress EEG signals. However, its performance is closely dependent on how a compression dictionary is built. Through our study, we notice that building the best fit over-complete Gabor dictionary plays an important role in this task. In this paper, we evaluate the effect of different time and frequency step sizes in building Gabor atoms on the performance of EEG signal compression using CS with three common EEG databases used by the research community. Taking the Normalized Mean Square Error (NMSE) as a performance metric, we present a quantitative study with an attempt to provide more insight on how to adopt CS in EEG signal compression.
\end{abstract}

\section{INTRODUCTION}

Electroencephalogram (EEG) is the recording of electrical activities of the brain through electrode sensors placed on the scalp, and was first introduced in 1924 by a German physiologist and psychiatrist Han Berger. Since measuring an EEG signal is a non-invasive procedure, it has been widely used as a clinical routine to diagnose physical or mental health problems such as epilepsy, loss of consciousness or dementia and sleep disorders. Moreover, EEG signals can be applied in construction of a Brain-Computer-Interface (BCI) to assist disabled people with daily living tasks. Nowadays, with the help of a modern wearable device, EEG signals could be uninterruptedly recorded for up to 14 days. This fact has promoted the widespread use of EEG applications such as Ambulance EEG monitoring (AEEG), telemedicine and provided patients with a flexible and cost-effective way to monitor their health in real-time [1-4].

In general, EEG signals are recorded using the 10-20 international system with the electrode placed first at $10 \%$, followed by four-section of $20 \%$ then ending with $10 \%$ of the total distance from the nasion to the inion; left to right preauricular point as describe in Fig. 1. Typically, 21 channels are recorded. For some special applications, it is possible to extend this 10-20 system by placing electrodes in between resulting in 32, 64, 128 and even 256 channels. With the sampling rate varying from $256 \mathrm{~Hz}$ to even $20 \mathrm{kHz}$ and the resolution up to 24bit, a second of EEG signal is approximately one kilobyte. As a result, the EEG data volume can easily exceed 10GB per day for a single patient. Moreover, a long-term (up to several months) recording of EEG signals that is required especially in the diagnosis of epilepsy produces a huge amount of data [5].

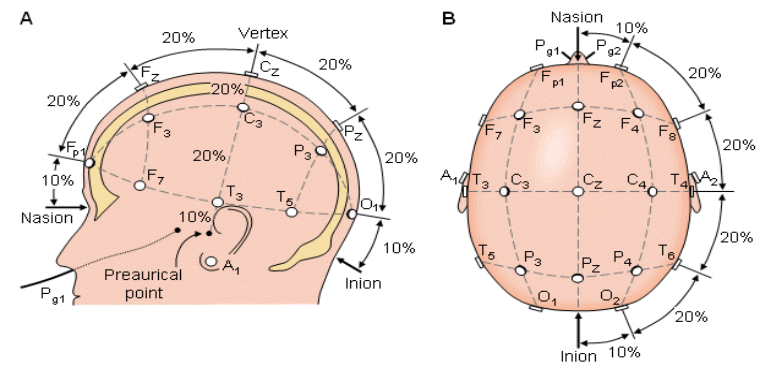

Fig. 1. EEG 10-20 system [1]

The problem of huge amount of data makes it impossible to store as well as monitor the EEG signals in real-time and realize prospective telemedicine systems. An obvious solution to this problem is to apply data compression and reduce the size of an EEG data file, which essentially involves trade-offs among various factors, including the compression ratio, the amount of distortion introduced, and the computational resources required to compress and reconstruct the data.

Compressed sensing (CS) [6-10] techniques have emerged with potential in the field of biomedical signal compression because they allow higher compression rates at a level of acceptable distortion, more importantly, with lower power consumption. With the benefit of minimizing the data volume and energy utilization in the sensor node, CS is best fitted to the context of a mobile, wearable and tele-healthcare system.

The fundamental idea behind CS is that we can represent signals using only a few non-zero coefficients in a suitable dictionary. As a result, the compression stage is simply the multiplication of the source signal with a sparse sensing matrix. The complexity in a CS system occurs in the reconstruction stage. Since the number of compressive measurements taken is far smaller than the number of samples in the original signal, the task of converting the compressed signal back into original one involves solving an underdetermined matrix equation. However, adding the constraint that the initial signal is sparse enables one to solve this underdetermined system of linear equations through convex optimization techniques $[11,12]$.

It is not trivial to find a suitable dictionary in CS, especially for a signal that is not really sparse in the time domain or transform domain, like an EEG signal. We aim to identify a suitable dictionary that is able to sparsely represent the signal before applying the optimization solution. The sparsity of EEG 
signal has been shown in Gabor domain [2, 3]. Parameterization of the time and frequency steps of Gabor atoms is an important factor in creating a best fit dictionary. In this paper, we evaluate the effect of different time and frequency step sizes in building Gabor atoms on the performance of EEG signal compression using CS with three common EEG databases used by the research community.

\section{EEG COMPRESSED SENSING}

Compressed sensing is based on the fundamental fact that we can represent many signals using only a few non-zero coefficients in a suitable dictionary. Fig. 2 shows the block diagram of a typical compressed sensing algorithm.

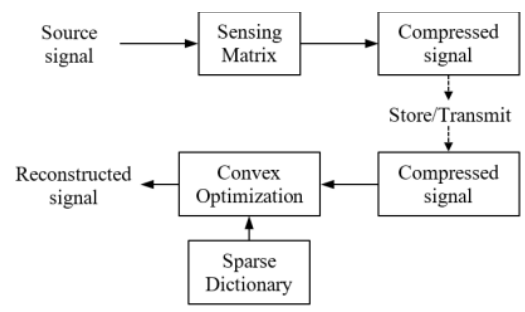

Fig. 2. Compressed sensing

\section{A. Compression}

Given a signal $\boldsymbol{x} \in \mathbb{R}^{N \times 1}$ is sparse, compressed sensing compresses it by a random matrix $\boldsymbol{\Phi} \in \mathbb{R}^{M \times N}$ :

$$
\mathbf{y}=\boldsymbol{\Phi} \mathbf{x}
$$

where $\boldsymbol{x}$ is the input signal, $\boldsymbol{\Phi}$ is the sampling matrix, and $\boldsymbol{y}$ is the compressed signal containing $M$ measurements. The compression is achieved by setting $M \ll N$. The measurement matrix $\boldsymbol{\Phi}$ must be maximally incoherent with the chosen dictionary. Previous works have shown that random matrices such as Gaussian, Bernoulli and sparse binary sensing matrices satisfy this requirement [2, 4-6].

Given that the input signal is sparse in the dictionary $\boldsymbol{D} \in$ $\mathbb{R}^{N \times P}$, we can represent the signal as:

$$
\mathbf{x}=\mathbf{D} \boldsymbol{\theta}=\sum_{i=1}^{K} \theta_{i} d_{i}
$$

With $K$ being the number of non-zero elements in the coefficient vector $\boldsymbol{\theta}$, the signal $\boldsymbol{x}$ can be represented by a few atoms $\boldsymbol{d}_{\boldsymbol{i}}$ from the dictionary. Thus, the compressed signal is

$$
y=\Phi x=\Phi D \theta
$$

\section{B. Reconstruction}

Given the compressed signal $\boldsymbol{y}$, the reconstruction stage needs to find $\boldsymbol{x}$ in order to satisfy (3). As the length of the unknowns $\boldsymbol{x}$ and $\boldsymbol{\theta}$ is much larger than the length of $\boldsymbol{y}$, there are infinitely many solutions for this equation.

The classical solution for this type of inverse problem is to minimize the Euclidean norm of the approximate signal $\widehat{\boldsymbol{x}}$ subject to satisfying that the product of the measurement matrix $\boldsymbol{\Phi}$ with the reconstructed signal is equal to the compressed signal $\boldsymbol{y}$. This solution is called least squares minimization or $\ell_{2}$ norm minimization:

$$
\widetilde{\boldsymbol{x}}=\operatorname{argmin}\|\widehat{\boldsymbol{x}}\|_{2} \text { subject to } \boldsymbol{\Phi} \widehat{\boldsymbol{x}}=\boldsymbol{y}
$$

Although (4) has a convenient closed form solution given by the normal equation, the solution is almost never sparse, resulting in a high reconstruction error.

As the $\ell_{2}$ norm minimization does not usually return a sparse vector, alternatives have been sought. One is to directly enforce a sparsity constraint on the solution, namely the $\ell_{0}$ norm, to minimize the number of non-zero elements in $\widehat{\boldsymbol{x}}$.

$$
\widetilde{\boldsymbol{x}}=\operatorname{argmin}\|\widehat{\boldsymbol{x}}\|_{0} \text { subject to } \boldsymbol{\Phi} \widehat{\boldsymbol{x}}=\boldsymbol{y}
$$

However, this is computationally unfeasible as it is NP hard. The solution is to relax (5) and use the $\ell_{1}$ norm, thereby minimizing the magnitude of the non-zero elements in $\widehat{x}$,

$$
\widetilde{\boldsymbol{x}}=\operatorname{argmin}\|\widehat{\boldsymbol{x}}\|_{1} \text { subject to } \boldsymbol{\Phi} \widehat{\boldsymbol{x}}=\boldsymbol{y}
$$

The $\ell_{1}$ norm has been proved to achieve a unique solution under certain conditions. As a result, $\boldsymbol{x}$ can be reconstructed exactly from compressed signal $\boldsymbol{y}$. With the constraint that $\boldsymbol{x}$ is sparse and the knowledge of the dictionary $\mathbf{D}$, the solution of $\boldsymbol{x}$ is equivalent to finding the best fit for the coefficient vector $\boldsymbol{\theta}$. As the result, the problem now becomes minimizing the magnitude of the non-zero elements in $\boldsymbol{\theta}$

$$
\min \|\boldsymbol{\theta}\|_{1} \text { subject to } \boldsymbol{y}=\boldsymbol{\Phi D} \boldsymbol{\theta}
$$

\section{Gabor Dictionary}

The real discrete time-frequency Gabor dictionary can be constructed by [7]:

$$
G\left(x, f, n_{0}, s, \varphi\right)=\exp \left(-\frac{\left(x-n_{0}\right)^{2}}{2 s^{2}}\right) \cos \left(2 \pi f\left(x-n_{0}\right)+\varphi\right)
$$

Fig. 3 is a plot of real Gabor atom of length $N=512$ at sampling rate of $f_{s}=128 \mathrm{~Hz}$ (solid line) with its Gaussian boundary (dashed line). The atom is centered at $n_{0}=256$, scale $s=64$, with frequency $f=4 \mathrm{~Hz}$ and phase $\varphi=0$.

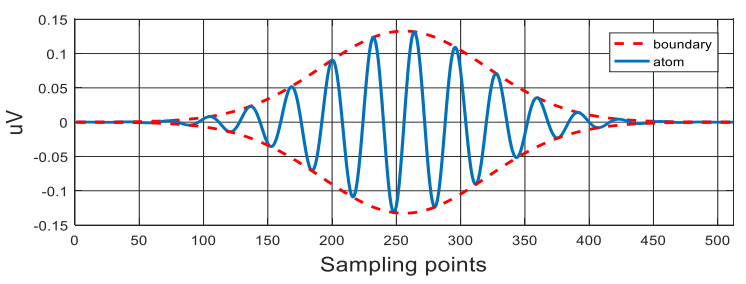

Fig. 3. Gabor atom

The time and frequency steps of such atoms play an important role in the reconstruction stage. Fig. 4 presents a simple example of representing a signal $A+B+C$ (plot 4) with $s=[16,32,64], \quad n_{0}=[32,160,384]$ and $f=$ $[12,16,8]$ are the scales, time shifts and frequencies of $\mathrm{A}, \mathrm{B}$ and $\mathrm{C}$, respectively.

The input EEG signal must first be epoched into nonoverlapping segments with a length of 4 seconds. Secondly, each segment will be resampled to $128 \mathrm{~Hz}$ to provide a realistic sampling rate in telemedicine applications. This step guarantees the uniformity of all datasets that we use in our work. Mean removal is also be applied to improve the compression ratio. This preprocessing step results with nonoverlapping EEG segments of length $N=512$. 


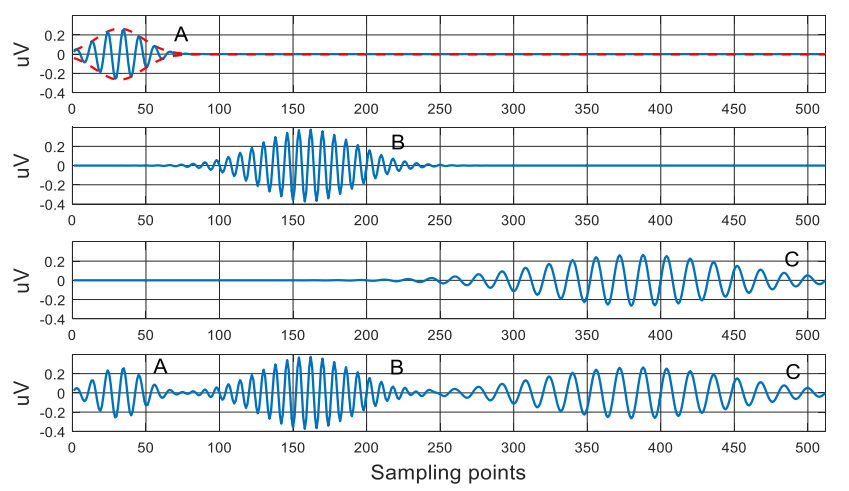

Fig. 4. Signal representation

As aforementioned, a random matrix ensures the maximal incoherent characteristics of the EEG signal without the knowledge of dictionary. We selected the white-noise Gaussian random matrix [8] as our sensing matrix to save the computation cost.

Gabor dictionary include atoms are parameterized as [6]:

$$
g_{\left(n, n_{0}, f_{0}, s\right)}=K_{\left(n_{0}, f_{0}, s\right)} \exp \left(-\frac{\left(n-n_{0}\right)^{2}}{2 s^{2}}\right) \sin \left(2 \pi f_{0}\left(n-n_{0}\right)\right)
$$

in which $s$ is the scale of atom, $n_{0}$ and $f_{0}$ are the center of atom, $K\left(n_{0}, f_{0}, s\right)$ is the normalization factor. Following the work in [6], we selected the time and frequency step as

$$
\Delta f_{0}=\sqrt{8 \pi \alpha} / s B, \Delta n_{0}=s B \sqrt{2 \alpha / \pi}
$$

with $\alpha=0.5 \ln (0.5(B+1 / B))$ and $B=2$ is the base.

The solution for the problem stated in (7) can be solved by setting the normal equation:

$$
\begin{aligned}
& \text { Set: } \mathbf{T}=\mathbf{\Phi D} \quad \Rightarrow \quad \mathbf{y}=\mathbf{T} \boldsymbol{\theta} \quad\left(\mathbf{T} \in \mathbb{R}^{\mathrm{M} \times \mathrm{P}}\right) \\
& \Leftrightarrow \mathbf{T}^{\prime} \mathbf{T} \boldsymbol{\theta}=\mathbf{T}^{\prime} \mathbf{y} \\
& \Leftrightarrow\left(\mathbf{T}^{\prime} \mathbf{T}\right)^{-1} \mathbf{T}^{\prime} \mathbf{T} \boldsymbol{\theta}=\left(\mathbf{T}^{\prime} \mathbf{T}\right)^{-1} \mathbf{T}^{\prime} \mathbf{y} \\
& \Leftrightarrow \boldsymbol{\theta}=\left(\mathbf{T}^{\prime} \mathbf{T}\right)^{-\mathbf{1}} \mathbf{T}^{\prime} \mathbf{y}
\end{aligned}
$$

Numerical optimization techniques such as Basis Pursuit (BP) Matching Pursuit (MP) of Orthogonal Matching Pursuit (OMP) are widely used to solve the reconstruction problem [4, 9-11]. In this work, we use a convex optimization method namely BP Denoising (BPDN) from the SPGL1 package [12].

\section{Experiment Setup}

To evaluate the effect of the time and frequency steps of the Gabor atoms in a wide range of EEG signals, we worked with three popular databases. The first two databases come from Physionet [13]. The first one was collected at the Children's Hospital Boston from 24 paediatric subjects with intractable seizures (CHB-MIT), and contains 664 files. The time recorded for each file is from 10 minutes up to 4 hours with a well-defined seizure notation. Data was collected at a sampling frequency of $256 \mathrm{~Hz}$, with a resolution of 16 bits per sample, and using up to 23 channels. The second one is a set of 108 polysomnographic recordings registered at the Sleep Disorders Center of the Ospedale Maggiore of Parma, Italy. With the recording time up to 10 hours each, this is a big database at sampling rate of $256 \mathrm{~Hz}$, resolution of 16 bits and from 5 to 13 channels.
The last database is from BCI Competition IV - dataset 1 [14], consisting of 7 files with 59 channels each, a sample rate of $1 \mathrm{KHz}$, and a resolution of 16 bits. The data was collected from healthy subjects with the EEG signals corresponding to a specific mental state.

With the total size from $7 \mathrm{~GB}$ up to $43 \mathrm{~GB}$ each, it is impractical to test the performance using the whole databases. As not all of the EEG channels from the database are actually EEG signals, and there are some dead channels with $\mathrm{NaN}$ or all zero values, we first had to remove these unusable channels from the databases. We had built the dataset with 100 randomly selected segments of 4 seconds from each database. To ensure uniformity across the datasets, we resampled the data to $128 \mathrm{~Hz}$.

\begin{tabular}{|c|c|c|c|c|c|c|c|}
\hline Scale & 1 & & & & & & \\
\hline$t_{f}=1$ & 2.13 & 4.26 & 8.53 & 1706 & 3411 & 6823 & 13645 \\
\hline$t_{f}=2$ & 1.07 & 2.13 & 4.26 & 8.53 & 17.06 & 34.11 & 68.23 \\
\hline$t_{f}=4$ & 0.53 & 1.07 & 2.13 & 4.26 & 8.53 & 17.06 & 34.11 \\
\hline$f_{f}=1$ & 53.59 & 26.79 & 13.40 & 6.70 & 3.35 & 1.67 & 0.84 \\
\hline$f_{f}=2$ & 26.79 & 13.40 & 6.70 & 3.35 & 1.67 & 0.84 & 0.42 \\
\hline$f_{f}=4$ & 13.40 & 6.70 & 3.35 & 1.67 & 0.84 & 0.42 & 0.21 \\
\hline$f_{f}=8$ & 6.70 & 3.35 & 1.67 & 0.84 & 0.42 & 0.21 & 0.01 \\
\hline
\end{tabular}

Based on the standard time and frequency steps described in (10), we define time and frequency factors, $t_{f}$ and $f_{f}$ then build a set of 12 dictionaries namely $\mathrm{D} t_{f} f_{f}$ with time step $t_{s}=$ $4 \Delta n_{0} / t_{f}$ (points) and frequency step $f_{s}=\Delta f_{0} / f_{f}(\mathrm{~Hz})$. Table I shows the time and frequency step at each scale.

Thus, as $t_{f}$ and $f_{f}$ increase, we obtain a higher resolution in time and frequency, resulting in larger dictionaries. Table II shows the size of each Gabor dictionary.

TABLE II. DICTIONARIES SIZE

\begin{tabular}{|c|c|c|c|c|c|}
\hline Name & \# atoms & Name & \# atoms & Name & \# atoms \\
\hline D11 & 1,918 & D21 & 3,801 & D41 & 7,529 \\
\hline D12 & 3,928 & D22 & 7,784 & D42 & 15,420 \\
\hline D14 & 8,042 & D24 & 15,938 & D44 & 31,578 \\
\hline D18 & 16,458 & D28 & 32,622 & D48 & 64,645 \\
\hline
\end{tabular}

To evaluate the compression achieved, we used the compression ratio (CR) defined as $\mathrm{CR}=N / M$, where $M$ is the number of measurements and $N$ is the length of input signal. The performance of the reconstruction stage will be measured by the normalized mean square error (NMSE) as $\operatorname{NMSE}(\mathbf{x}, \hat{\mathbf{x}})=\|\mathbf{x}-\hat{\mathbf{x}}\|^{2} /\left\|\mathbf{x}-\boldsymbol{\mu}_{\mathbf{x}}\right\|^{2}$, where $\boldsymbol{x}$ is the original signal, $\widehat{\boldsymbol{x}}$ is the reconstructed signal and $\mu_{\boldsymbol{x}}$ is the mean of $\boldsymbol{x}$.

\section{RESUlTS AND DISCUSSION}

The NMSE of the reconstruction for each dictionary over the three datasets is shown in Table III. There a few points to note here: (i) As the CR decreases, the NMSE decreases. This is to be expected, due to more signal information being available to the reconstruction algorithm. (ii) As the dictionary size increases - due to either increased time or frequency resolution - the NMSE decreases. This is due to the dictionary being able to more accurately represent the signal, as in (3). (iii) These trends are consistent across the datasets, which is intuitively satisfying. Figs. 5 and 6 present the NMSE of the reconstruction dictionaries averaged across the three datasets. Fig. 5 shows the mean NMSE in descending order. Despite the fact that D28 is better in term of reconstruction rate than D24 
most of the time, the D24 dictionary seems to be the best choice for reconstruction performance whilst minimizing the dictionary size (half the size of D28), given that the dictionary size will impact memory requirements and computation cost. Fig. 6 illustrates that although the increased dictionary size improves the NMSE, the effect has diminishing returns, particularly above a dictionary size of 16,000 .

\begin{tabular}{|c|c|c|c|c|}
\hline \multicolumn{2}{|c|}{ CR } & \multirow[t]{2}{*}{8} & \multirow[t]{2}{*}{4} & \multirow[t]{2}{*}{2} \\
\hline Dataset & Dictionary & & & \\
\hline \multirow{12}{*}{ CHB-MIT } & D11 & 1.0638 & 0.9380 & 0.7412 \\
\hline & D12 & 0.8722 & 0.5568 & 0.2087 \\
\hline & D14 & 0.7518 & 0.3992 & 0.1398 \\
\hline & D18 & 0.7403 & 0.3837 & 0.1356 \\
\hline & D21 & 1.0182 & 0.7707 & 0.5660 \\
\hline & D22 & 0.7851 & 0.4240 & 0.1450 \\
\hline & D24 & 0.6934 & 0.3656 & 0.1270 \\
\hline & D28 & 0.6903 & 0.3802 & 0.1223 \\
\hline & D41 & 0.9289 & 0.6376 & 0.3310 \\
\hline & D42 & 0.7355 & 0.4018 & 0.1300 \\
\hline & D44 & 0.6928 & 0.3826 & 0.1267 \\
\hline & $\mathrm{D} 48$ & 0.6830 & 0.3566 & 0.1236 \\
\hline \multirow{12}{*}{ CAP } & D11 & 1.1472 & 1.0808 & 1.0804 \\
\hline & D12 & 0.8102 & 0.4418 & 0.1365 \\
\hline & D14 & 0.5264 & 0.2427 & 0.0633 \\
\hline & D18 & 0.5207 & 0.2273 & 0.0579 \\
\hline & D21 & 1.0766 & 0.9375 & 0.8525 \\
\hline & D22 & 0.5756 & 0.2578 & 0.0666 \\
\hline & D24 & 0.4705 & 0.2064 & 0.0514 \\
\hline & D28 & 0.4557 & 0.2095 & 0.0522 \\
\hline & D41 & 0.9518 & 0.7928 & 0.6291 \\
\hline & D42 & 0.5777 & 0.2457 & 0.0635 \\
\hline & D44 & 0.5128 & 0.2133 & 0.0520 \\
\hline & $\mathrm{D} 48$ & 0.4689 & 0.2219 & 0.0527 \\
\hline \multirow{12}{*}{ BCI } & D11 & 1.0980 & 1.0446 & 1.0705 \\
\hline & D12 & 0.7690 & 0.4367 & 0.1531 \\
\hline & D14 & 0.4908 & 0.2306 & 0.0823 \\
\hline & D18 & 0.4498 & 0.2249 & 0.0800 \\
\hline & D21 & 1.0522 & 0.9311 & 0.8844 \\
\hline & D22 & 0.6118 & 0.2722 & 0.0949 \\
\hline & D24 & 0.4352 & 0.2038 & 0.0777 \\
\hline & D28 & 0.4281 & 0.2038 & 0.0740 \\
\hline & D41 & 0.9755 & 0.8108 & 0.6741 \\
\hline & D42 & 0.5531 & 0.2477 & 0.0870 \\
\hline & D44 & 0.4350 & 0.2092 & 0.0742 \\
\hline & D48 & 0.4202 & 0.2026 & 0.0728 \\
\hline
\end{tabular}

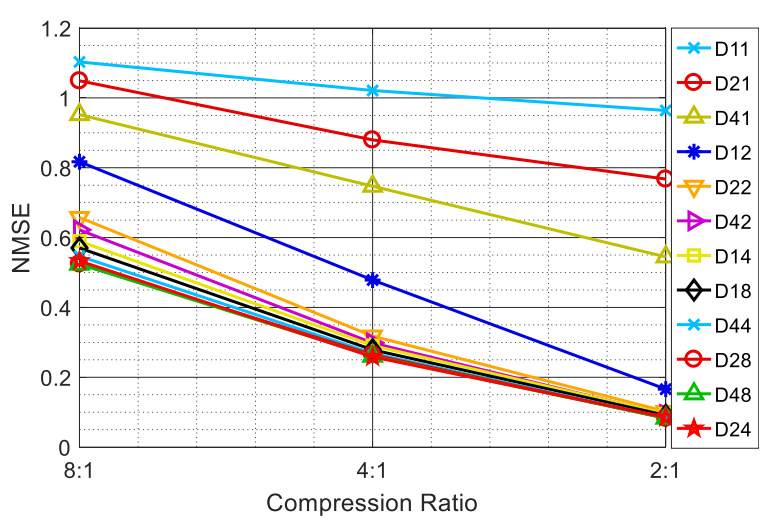

Fig. 5. Mean NMSE for different dictionaries

\section{CONCLUSION}

This paper presented a study into the effect of Gabor atom dictionaries' time and frequency resolution in compressed sensing of EEG signals. The results showed that a dictionary with about 16,000 offers an excellent tradeoff when comparing reconstruction performance with memory requirements and reconstruction time. Future work includes investigating whether further improvements can be gained by tailoring the Gabor dictionary to specific EEG characteristics.
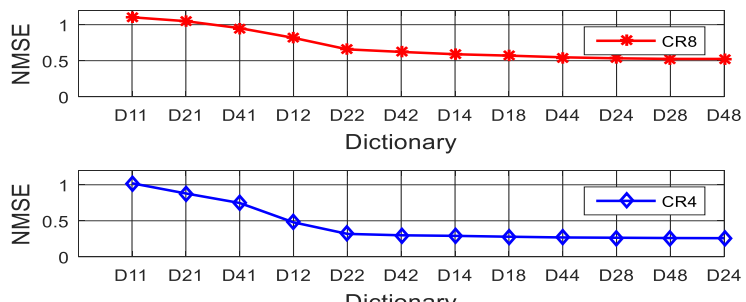
Dictionary

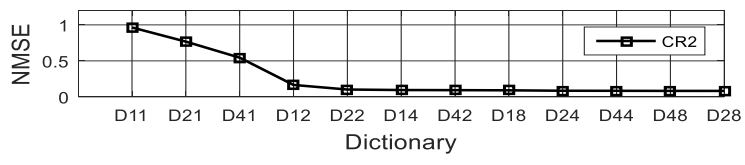

Fig. 6. Mean NMSE at each compression ratio

\section{REFERENCES}

[1] Human electroencephalography. Available: http://tktamop.elte.hu/ online-tananyagok/physiology_practical/ch10s02.html

[2] M. Mohsina and A. Majumdar, "Gabor based analysis prior formulation for EEG signal reconstruction," Biomedical Signal Processing and Control, vol. 8, pp. 951-955, 2013.

[3] S. Aviyente, "Compressed Sensing Framework for EEG Compression," in Proc. of IEEE SSP '07, pp. 181-184.

[4] A. Abdulghani, A. Casson, and E. Rodriguez-Villegas, "Compressive sensing scalp EEG signals: implementations and practical performance," Medical \& Biological Engineering \& Computing, vol. 50, pp. 1137-1145, 2012.

[5] Z. Zhilin, J. Tzyy-Ping, S. Makeig, and B. D. Rao, "Compressed Sensing of EEG for Wireless Telemonitoring With Low Energy Consumption and Inexpensive Hardware," IEEE Transactions on Biomedical Engineering, vol. 60, pp. 221-224, 2013.

[6] S. Fauvel and R. K. Ward, "An energy efficient compressed sensing framework for the compression of electroencephalogram signals," Sensors, vol. 14, pp. 1474-1496, 2014.

[7] M. Barwiński, Product-Based Metric for Gabor Functions and Its Implications for the Matching Pursuit Analysis. University of Warsaw, Warsaw, Poland: University of Warsaw, Warsaw, Poland, 2004.

[8] D. Craven, B. McGinley, L. Kilmartin, M. Glavin, and E. Jones, "Compressed Sensing for Bioelectric Signals: A Review," IEEE Jrnl. of Biomedical and Health Informatics, vol. 19, pp. 529-540, 2015.

[9] S. S. Chen, D. L. Donoho, and M. A. Saunders, "Atomic Decomposition by Basis Pursuit," SIAM Journal on Scientific Computing, vol. 20, pp. 33-61, 1998.

[10] S. G. Mallat and Z. Zhang, "Matching pursuits with time-frequency dictionaries," Signal Processing, IEEE Transactions on, vol. 41, pp. 3397-3415, 1993.

[11] H. Mamaghanian, N. Khaled, D. Atienza, and P. Vandergheynst, "Compressed Sensing for Real-Time Energy-Efficient ECG Compression on Wireless Body Sensor Nodes," IEEE Transactions on Biomedical Engineering, vol. 58, pp. 2456-2466, 2011.

[12] E. v. d. Berg and M. P. Friedlander, "Probing the Pareto Frontier for Basis Pursuit Solutions," SIAM Journal on Scientific Computing, vol. 31, pp. 890-912, 2009.

[13] A. L. Goldberger, et al., "PhysioBank, PhysioToolkit, and PhysioNet component of a new research resource for complex physiologic signals," Circulation Electronic Pages, 2002.

[14] B. Blankertz, F. Losch, M. Krauledat, G. Dornhege, G. Curio, M. K. R, et al., "The Berlin Brain-Computer Interface: Accurate performance from first-session in BCI-naive subjects," IEEE Transactions on Biomedical Engineering, vol. 55, pp. 2452-2462, 2008. 